CrossMark

\title{
Adenotonsillectomy for childhood obstructive sleep apnoea reduces thoraco-abdominal asynchrony but spontaneous apnoea-hypopnoea index normalisation does not
}

\author{
Xiao Liu', Sarah Immanuel ${ }^{1}$, Yvonne Pamula² $^{2}$ Declan Kennedy ${ }^{2,3}$, \\ James Martin ${ }^{2}$ and Mathias Baumert ${ }^{1}$
}

Affiliations: 'School of Electrical and Electronic Engineering. The University of Adelaide, Adelaide, Australia. ${ }^{2}$ Dept of Respiratory and Sleep Medicine, Women's and Children's Hospital, Adelaide, Australia. ${ }^{3}$ Children's Research Centre, School of Paediatrics and Reproductive Health, The University of Adelaide, Adelaide, Australia.

Correspondence: Mathias Baumert, School of Electrical and Electronic Engineering, The University of Adelaide, Adelaide, SA 5005, Australia. E-mail: mathias.baumert囚adelaide.edu.au

@ERSpublications

Adenotonsillectomy for childhood obstructive sleep apnoea reduces respiratory effort during sleep http://ow.ly/TJcx304lKdm

Cite this article as: Liu X, Immanuel S, Pamula Y, et al. Adenotonsillectomy for childhood obstructive sleep apnoea reduces thoraco-abdominal asynchrony but spontaneous apnoea-hypopnoea index normalisation does not. Eur Respir J 2017; 49: 1601177 [https://doi.org/10.1183/13993003.01177-2016].

ABSTRACT The efficacy of adenotonsillectomy for treating obstructive sleep apnoea syndrome (OSAS) in children has been established, but its precise effects on inspiratory effort are not well documented.

In 353 children enrolled in the Childhood Adenotonsillectomy Trial, randomised to undergo either early adenotonsillectomy $(\mathrm{n}=182)$ or a strategy of watchful waiting with supportive care (WWSC) $(\mathrm{n}=171)$, thoraco-abdominal asynchrony (TAA) was analysed during quiet, non-apnoeic and non-hypopnoeic breathing during sleep at baseline and at 7 months using overnight polysomnography.

Children who underwent early adenotonsillectomy demonstrated a reduction in TAA post-surgery while the WWSC arm showed no change. On assessing TAA with regard to normalisation of clinical polysomnography findings at follow-up, TAA was reduced in children who had surgical resolution of OSAS (based on apnoea-hypopnoea index), but not in children who displayed spontaneous normalisation of apnoea-hypopnoea index. In the latter group, TAA was inversely correlated with quality of life.

We conclude that adenotonsillectomy reduces TAA during quiet sleep. Monitoring of instantaneous TAA may yield additional insight in the dynamic changes of inspiratory effort. In combination with traditional indices of obstruction, TAA may more accurately characterise the degree of sleep-disordered breathing in children.

This article has supplementary material available from erj.ersjournals.com

Received: June 142016 | Accepted after revision: Sept 142016

This study was registered at clinicaltrials.gov with identifier number NCT00560859.

Support statement: This project was partly supported by grant ARC DP 110102049. This CHAT study was supported by grants (HL083075, HL083129, UL1-RR-024134, UL1 RR024989) from the National Institutes of Health. Funding information for this article has been deposited with the Open Funder Registry.

Conflict of interest: None declared.

Copyright OERS 2017 


\section{Introduction}

Upper airway obstruction during sleep is relatively common during childhood, with a reported prevalence between 3 and 15\% [1]. Its severity ranges from primary snoring to obstructive sleep apnoea syndrome (OSAS), with the majority of children showing symptoms at the milder end of the spectrum. While clinical concerns have focused largely on the associated behavioural and cognitive deficits, mounting evidence suggests that OSAS during childhood also affects the cardiovascular system, which if untreated may develop into cardiovascular disease later in life [2,3].

In otherwise normal children, OSAS is most frequently observed when the tonsils and adenoids are enlarged and a family history of OSAS exists. It is characterised by increased upper airway collapsibility and upper airway loading. Consequently, adenotonsillectomy (AT) is commonly the first line of treatment. The efficacy of AT in treating the range of adverse health outcomes reported in children with OSAS, particularly for milder OSAS, has remained largely untested. Additional concerns pertain to post-surgical complications and the healthcare costs of performing large numbers of ATs [4-6].

Clinically, the severity of OSAS is assessed by using overnight polysomnography (PSG) and observing the rate of respiratory events (apnoea and hypopnoea). Although the limitations of simple indices derived from discretely scored events, such as the apnoea-hypopnoea index (AHI), have been debated within the sleep community, they are considered useful in current clinical practice $[7,8]$. However, since the frequency of respiratory events is low in children with mild OSAS, the AHI may not reflect the overall impact on respiratory loading. Thus, other PSG measures that quantify inspiratory effort might add a useful dimension to the assessment of breathing disturbance. While AT has been shown to effectively reduce the number of incidents of apnoea and hypopnoea, it is less clear cut whether it also reduces inspiratory effort during respiratory-event-free periods of sleep [9].

The aim of this study was to investigate the effects of AT for treatment of OSAS on an indirect marker of inspiratory effort, namely the phase shift between thoracic and abdominal movements (thoraco-abdominal asynchrony (TAA)), by utilising data from the Childhood Adenotonsillectomy Trial (CHAT). The CHAT study is a landmark multicentre controlled trial evaluating health and behavioural outcomes in children with OSAS randomised into early AT (eAT) or watchful waiting with supportive care (WWSC) [10, 11]. We hypothesised that AT reduces inspiratory effort throughout respiratory-event-free sleep and thereby reduces TAA.

\section{Methods}

Study sample

Detailed particulars of the CHAT protocol have been published [11]. Data are publicly available at https:// sleepdata.org/datasets/chat. Children between 5.0 and 9.9 years of age with PSG-confirmed OSAS (i.e. obstructive $\mathrm{AHI} \geqslant 2$ events. $\mathrm{h}^{-1}$ or an obstructive apnoea index $(\mathrm{OAI}) \geqslant 1$ events $\cdot \mathrm{h}^{-1}$ ), a history of snoring and considered to be surgical candidates for AT were recruited from paediatric sleep centres/sleep laboratories, paediatric otolaryngology clinics, general paediatric clinics and the general community from six clinical centres. Exclusion criteria included comorbidities, medications for psychiatric or behavioural

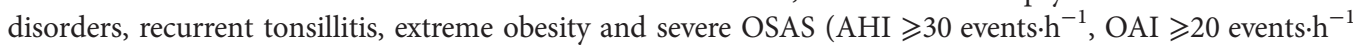
or oxyhaemoglobin saturation $<90 \%$ for $>2 \%$ of total sleep time). The study was approved by the Institutional Review Board of each institution. Informed consent was obtained from caregivers, and assent from children $\geqslant 7$ years of age. The study was registered at Clinicaltrials.gov (\#NCT00560859).

\section{CHAT interventions}

Children were randomly assigned to either eAT (surgery within 4 weeks after randomisation) or a strategy of WWSC with reassessment of all the study variables at approximately 7 months. Complete bilateral tonsillectomy and removal of obstructing adenoid tissue was performed using standard surgical techniques.

\section{Overnight polysomnography}

Each child underwent in-laboratory baseline and follow-up PSG carried out by study-certified technicians, following American Academy of Sleep Medicine paediatric guidelines for both acquisition and scoring [12]. The PSGs were centrally scored by registered sleep technicians. Overnight PSG was repeated approximately 7 months after randomisation $[11,13]$.

\section{Analysis of thoraco-abdominal asynchrony}

PSG recordings of ribcage and abdominal inductance belts were utilised to measure the instantaneous phase difference between thoracic and abdominal excursions (TAA). For details, see supplementary material. Only portions of PSG that were free from discretely scored events (e.g. arousal, apnoea, hypopnoea, limb movement) and artefacts were included in the analysis. Instantaneous TAA values were averaged within each sleep stage. The mean \pm SD portion of sleep included in TAA analysis of the eAT arm 
was $76.8 \pm 12.9 \%$ at baseline and $86.5 \pm 7.68 \%$ at follow-up. In the WWSC arm, the sleep portions were $77.8 \pm 11.9 \%$ and $80.4 \pm 12.3 \%$, respectively (supplementary table S1). The TAA values for each recording can be obtained at https://sleepdata.org/datasets/chat

\section{Neurophysiological tests and surveys}

Neurophysiological tests and surveys were performed as part of the original CHAT study to assess behaviour, OSAS symptoms, sleepiness, quality of life and generalised intellectual functioning (supplementary material).

\section{Statistical analysis}

Anthropometric data were compared using t-tests and Chi-squared tests as appropriate. TAA values were log-transformed to achieve normal distribution and analysed for stages 2 (N2) and 3 (N3) in non-rapid eye movement (NREM) sleep as well as rapid eye movement (REM) sleep (R). One-way repeated measures ANOVA was performed to investigate the effect of sleep stage on TAA, followed by a Bonferroni test based on the t-statistic for post hoc comparison. Two-way ANCOVA was carried out to test the effect of surgery and time point (baseline versus follow-up; repeated measure) on TAA. Anthropometric variables that were likely to confound statistical analysis (body mass index (BMI) z-score, BMI z-score change between follow-up and baseline, age, sex and race) were included in the model as covariates. Subsequently, three-way ANCOVA was conducted to investigate the effects of AHI normalisation, study arm and time point on TAA. Spearman correlation analysis was performed to explore the relationship between AHI, the extent of oxygen desaturation, peak end-tidal carbon dioxide and TAA. To explore whether TAA has potential clinical value in stratifying OSAS diagnostics, we performed Spearman correlation analysis between TAA and previously reported measures of behaviour, OSAS symptom indicators, sleepiness, global quality of life and intellectual functioning [10] in those children whose AHI was normal during follow-up and hence OSAS was considered resolved.

\section{Results}

\section{Subject demographics}

In total, 353 children of the original CHAT study who underwent both baseline and follow-up PSG and whose respiratory inductance signals met the technical criteria were included in this study. Of these, 182 children underwent eAT and 171 children joined the WWSC group (figure 1). Both groups had comparable demographic profiles (table 1). The mean age of the participants at baseline was 6.6 years and 49\% were male. Approximately half (54\%) of the sample were African American and 34\% were obese. Around $5 \%$ of children were treated with montelukast and $\sim 22 \%$ received glucocorticoids for rhinitis or asthma at the time of the baseline PSG. At follow-up, $83 \%$ of children in the eAT arm no longer had AHI-defined OSAS, i.e. values of AHI $\leqslant 2$ and OAI $\leqslant 1$, while $40 \%$ of children in the WWSC arm had spontaneous normalisation of AHI scores. Approximately $7 \%$ of children in the eAT arm and $8 \%$ in the WWSC arm were on montelukast, and 24\% (eAT) and 26\% (WWSC) were on glucocorticoids at the time of the follow-up PSG, representing a small but statistically nonsignificant increase compared with baseline.

\section{Effect of sleep stage on TAA}

Sleep stage had a significant effect on TAA (reported and analysed as log-transformed values in degrees) (N2: $3.20 \pm 0.72 \log ^{\circ}, \mathrm{N} 3: 3.24 \pm 0.81 \log ^{\circ}$, R: $3.56 \pm 0.67 \log ^{\circ}$; $\mathrm{p}<0.001$; measured on baseline PSG). Post hoc analysis showed significantly higher TAA in REM sleep compared with both NREM sleep stages ( $\mathrm{R}$ versus N2: $\mathrm{p}<0.001$, $R$ versus $\mathrm{N} 3$ : $\mathrm{p}<0.001$ ), but no significant difference between NREM sleep stages (N2 versus $\mathrm{N} 3: \mathrm{p}=0.448$ ).

Due to the effect of sleep stage on TAA, all subsequent data analyses were performed separately for each sleep stage.

\section{Correlation between TAA and clinical measures of hypoxia and hypoventilation}

Using TAA values obtained from baseline and follow-up PSG, statistically significant but weak positive correlations with the extent of oxygen desaturation were observed (percentage of sleep time spent at oxygen saturation $\leqslant 90 \%, N 2$ : $r=0.179, p<0.001 ; N 3: r=0.135, p<0.001 ; R: r=0.172, p<0.001)$. A weak yet statistically significant positive correlation between TAA and the percentage of sleep time at a partial carbon dioxide pressure above $50 \mathrm{mmHg}$ (log-transformed) was observed in REM sleep ( $\mathrm{r}=0.082$, $\mathrm{p}<0.048$ ). TAA also showed a statistically significant but weak positive correlation with AHI across all three sleep stages (N2: $r=0.281, \mathrm{p}<0.001 ; \mathrm{N} 3: \mathrm{r}=0.251, \mathrm{p}<0.001$; $\mathrm{R}: \mathrm{r}=0.238, \mathrm{p}<0.001)$.

Effect of surgery on TAA

No significant difference in TAA between baseline and follow-up PSG was observed in any sleep stage (table 2). Significant study arm effects were observed in sleep stages N2 and N3. Time point×study arm interaction effects were significant across all stages of sleep, consistently pointing towards a reduction in 


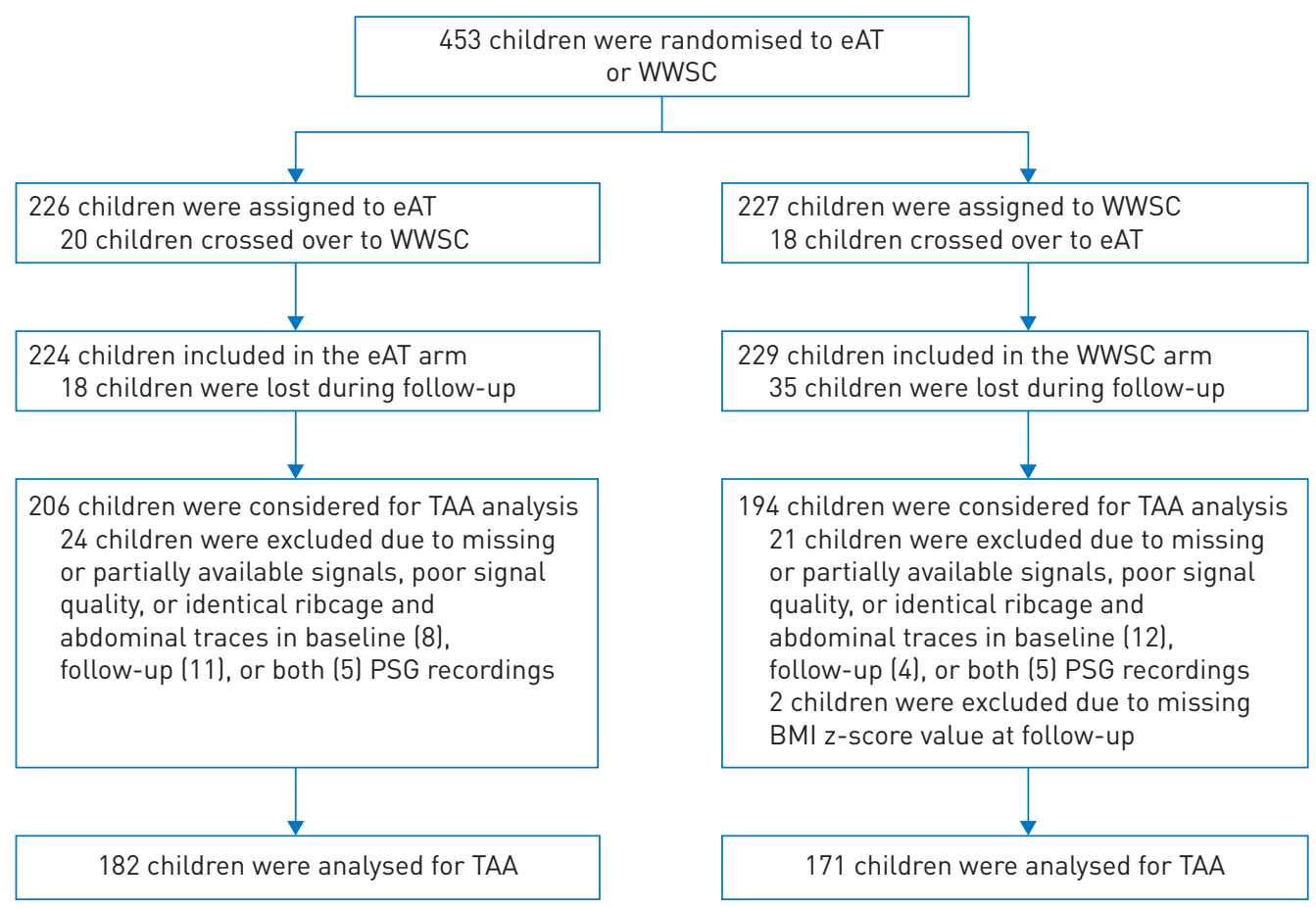

FIGURE 1 Summary of Childhood Adenotonsillectomy Trial study participants included in the thoraco-abdominal asynchrony (TAA) analysis. BMI: body mass index; eAT: early adenotonsillectomy; PSG: polysomnography; WWSC: watchful waiting with supportive care.

TAA in the eAT arm following AT. Post hoc comparison showed significantly lower TAA during follow-up PSG compared with baseline in the eAT arm only across all sleep stages $(\mathrm{N} 2$ : $\mathrm{p}<0.0001 ; \mathrm{N} 3$ : $\mathrm{p}<0.0001$; $\mathrm{R}: \mathrm{p}<0.0001)$. The eAT arm displayed significantly lower TAA than the WWSC arm during follow-up PSG throughout all sleep stages $(\mathrm{N} 2: \mathrm{p}<0.0001 ; \mathrm{N} 3: \mathrm{p}<0.0001 ; \mathrm{R}: \mathrm{p}=0.031)$. Of the covariates included in the model, increase in age was associated with a significant TAA reduction in REM sleep $(\mathrm{F}=8.73, \mathrm{p}=0.003)$. Sex showed a weak but significant association with TAA in sleep stage N2 $(F=3.99, p=0.046)$, where boys had lower TAA than girls.

TABLE 1 Baseline characteristics of subjects grouped according to study arm and apnoeahypopnoea index (AHI) normalisation at 7 months

\begin{tabular}{|c|c|c|c|c|}
\hline \multirow[t]{2}{*}{ Characteristic } & \multicolumn{2}{|c|}{ Study arm } & \multicolumn{2}{|c|}{$\mathrm{AHI}$ at 7 months } \\
\hline & eAT & WWSC & Normalised & Not normalised \\
\hline Subjects & 182 & 171 & 219 & 134 \\
\hline Age years & $6.64 \pm 1.46$ & $6.60 \pm 1.40$ & $7.10 \pm 1.46$ & $7.31 \pm 1.43$ \\
\hline Male n (\%) & $83(45.6 \%)$ & 89 (52\%) & $102(46.6 \%)$ & $70(52.2 \%)$ \\
\hline \multicolumn{5}{|l|}{ Race n $(\%)^{\#}$} \\
\hline African American & $95(52.2 \%)$ & $97(56.7 \%)$ & $105(47.9 \%)$ & $87(64.9 \%)$ \\
\hline Caucasian & $67(36.8 \%)$ & $59(34.5 \%)$ & $90(41.1 \%)$ & $36(26.9 \%)$ \\
\hline Other & $20(11 \%)$ & $15(8.8 \%)$ & $24(11 \%)$ & $11(8.2 \%)$ \\
\hline BMI z-score & $0.91 \pm 1.36$ & $0.86 \pm 1.26$ & $0.94 \pm 1.20$ & $1.38 \pm 1.25$ \\
\hline \multicolumn{5}{|l|}{ Weight class n $(\%)^{\Upsilon}$} \\
\hline Overweight & $92(50.5 \%)$ & $79(46.2 \%)$ & $104(47.5 \%)$ & $86(64.2 \%)$ \\
\hline Obese & $63(34.6 \%)$ & $58(33.9 \%)$ & $70(32 \%)$ & $68(50.7 \%)$ \\
\hline Montelukast n (\%) & $8(4.4 \%)$ & $9(5.3 \%)$ & $12(5.5 \%)$ & $15(11.2 \%)$ \\
\hline Glucocorticoids n (\%) & $40(22 \%)$ & $37(21.6 \%)$ & $52(23.7 \%)$ & $37(27.6 \%)$ \\
\hline
\end{tabular}

Data are presented as mean \pm SD unless otherwise stated. eAT: early adenotonsillectomy; WWSC: watchful waiting and supportive care; BMI: body mass index. " : reported by caregivers; ๆ: overweight was defined as $\mathrm{BMI} \geqslant 85$ th percentile, obese as $\mathrm{BMI} \geqslant 95$ th percentile. 
TABLE 2 Comparison of thoraco-abdominal asynchrony (TAA) between time points and study arm across sleep stages

\begin{tabular}{|c|c|c|c|c|c|c|c|}
\hline \multirow[t]{2}{*}{ Parameter } & \multicolumn{2}{|c|}{ eAT ( $n=182)$} & \multicolumn{2}{|c|}{ WWSC (n=171) } & \multicolumn{3}{|c|}{ p-value } \\
\hline & Baseline & Follow-up & Baseline & Follow-up & Time point & Study arm & Study arm $\times$ time point \\
\hline N2 TAA ${ }^{\#}$ (degree) & $3.23 \pm 0.72$ & $2.89 \pm 0.72$ & $3.17 \pm 0.72$ & $3.21 \pm 0.73$ & 0.326 & 0.025 & $<0.0001$ \\
\hline R TAA ${ }^{\#}$ (degree) & $3.62 \pm 0.70$ & $3.29 \pm 0.67$ & $3.49 \pm 0.63$ & $3.44 \pm 0.67$ & 0.687 & 0.739 & 0.0019 \\
\hline
\end{tabular}

Data are presented as group mean \pm SD. p-values were obtained by using two-way ANCOVA adjusted for likely confounding factors of age (5-9 years), race (black, white, other), body mass index (BMI) z-score, BMI z-score change and sex. eAT: early adenotonsillectomy; WWSC: watchful waiting with supportive care; N2: stage 2 non-rapid eye movement sleep; N3: stage 3 non-rapid eye movement sleep; R: rapid eye movement sleep. " : reported and analysed as log-transformed values.

\section{Effect of AHI normalisation and surgery on TAA}

No significant TAA differences between baseline and follow-up, between eAT and WWSC groups, or study arm $\times$ AHI normalisation interactions were observed in any of the sleep stages (table 3). AHI normalisation and time point $\times$ study arm $\times \mathrm{AHI}$ normalisation interactions were significant in stages $\mathrm{N} 2$ and N3. Study $\times$ AHI normalisation interaction effects were significant across all sleep stages, consistently pointing towards a reduction in TAA in the normalised group post-AT. However, the time point $\times$ study arm interaction effect was significant only within stage N2. Of the covariates included in the model, increase in age was associated with a significant TAA reduction in REM sleep ( $\mathrm{F}=8.99, \mathrm{p}=0.003)$. Sex showed a weak but significant association with TAA in sleep stage N2 $(\mathrm{F}=4.96, \mathrm{p}=0.027)$, where boys had lower TAA than girls.

When comparing baseline TAA with follow-up TAA for both arms (figure 2), significant differences were found only in the subgroup of children in the eAT arm whose AHI normalised during follow-up. This was consistent across all sleep stages $(\mathrm{N} 2$ : $\mathrm{p}<0.00001 ; \mathrm{N} 3$ : $\mathrm{p}<0.00001 ; \mathrm{R}: \mathrm{p}<0.00001)$.

Post hoc comparisons of the follow-up data showed a significant TAA decrease in children whose AHI normalised compared with children whose AHI remained abnormal (figure 3). This was observed within the WWSC and eAT arms across all sleep stages (WWSC: N2: $p=0.0078 ; \mathrm{N} 3: \mathrm{p}=0.0044 ; \mathrm{REM}$ : $\mathrm{p}=0.0254$; eAT: N2: $p=0.0007 ; \mathrm{N} 3: \mathrm{p}=0.0023$; $\mathrm{R}: \mathrm{p}=0.0200$ ) (figure 3 ). The TAA reduction was more pronounced in children of the eAT arm compared with the WWSC arm (N2: $\mathrm{p}=0.028)$.

\section{Correlation between TAA and cognitive, behavioural and OSAS symptom indicators in children with normal AHI}

Among those children that were classified as normal on clinical PSG score during follow-up, statistically significant negative correlations were observed between the parent total scale score of the Paediatric Quality of Life Inventory and TAA during NREM sleep (N2: $r=-0.183, p<0.01 ; \mathrm{N} 3: \mathrm{r}=-0.147, \mathrm{p}<0.05$ ), but not with AHI. In addition, the total obstructive sleep apnoea-18 (OSA-18) survey score was positively correlated with TAA during NREM sleep (N2: $\mathrm{r}=0.151, \mathrm{p}<0.05$; N3: $\mathrm{r}=0.149, \mathrm{p}<0.05)$, but not with AHI. No correlations were found between TAA and behaviour, OSAS symptom measures or sleepiness. When analysing the eAT and WWSC arms separately, correlations between the parent total scale score of the Paediatric Quality of Life Inventory and TAA were evident in the WWSC arm (N2: $r=-0.360, p<0.005$; N3: $\mathrm{r}=-0.314, \mathrm{p}<0.01 ; \mathrm{R}: \mathrm{r}=-0.246, \mathrm{p}<0.05$ ) (figure 4), but not in the eAT arm. Correlations between OSA-18 score and TAA were no longer significant.

\section{Discussion}

Our main finding is a reduction in TAA during quiet, event-free sleep in children with OSAS following AT, indicating an overall reduction in inspiratory effort. However, this was not observed in children whose OSAS resolved spontaneously (as measured by the clinical diagnostic marker, i.e. AHI). Outcome-specific analysis suggests that normalisation of AHI, in particular in those children who underwent AT, is associated with TAA reduction at 7-month follow-up. In children whose AHI normalised without surgical intervention and hence were clinically diagnosed as OSAS free at follow-up, high TAA values were associated with poorer quality of life. This indicates that increased inspiratory effort, even in the absence of frank apnoea or hypopnoea, has adverse health outcomes.

TAA measures the phase angle between thoracic and abdominal excursions and is considered a noninvasive measure of inspiratory effort [9]. We have recently devised a robust, fully automated method of TAA measurement that can be easily implemented in PSG analysis [14]. Although TAA cannot provide 
TABLE 3 Comparison of thoraco-abdominal asynchrony (TAA) between time points, study arm and apnoea-hypopnoea index (AHI) normalisation across sleep stages

Parameter

\begin{tabular}{|c|c|c|c|c|c|c|c|}
\hline \multicolumn{2}{|c|}{ eAT (n=182) } & \multicolumn{2}{|c|}{ WWSC (n=171) } & \multicolumn{4}{|r|}{$p$-value } \\
\hline $\begin{array}{c}\text { AHI } \\
\text { normalised } \\
(n=151)\end{array}$ & $\begin{array}{c}\text { AHI not } \\
\text { normalised } \\
\text { (n=31) }\end{array}$ & $\begin{array}{c}\text { AHI } \\
\text { normalised } \\
(n=68)\end{array}$ & $\begin{array}{c}\text { AHI not } \\
\text { normalised } \\
\text { (n=103) }\end{array}$ & Time point & Study arm & Normalisation & $\begin{array}{c}\text { Time } \\
\text { point } \times \text { study arm }\end{array}$ \\
\hline
\end{tabular}

( $n=31$ )

( $n=68$ )

( $n=103$ )

$\begin{array}{cc}\text { Study } & \text { Time } \\ \text { arm } \times \text { AHI } & \text { point } \times \text { AHI } \\ \text { normalisation } & \text { normalisation }\end{array}$

Study

normalisation normalisation arm×time

normalisation

\begin{tabular}{|c|c|c|c|c|c|c|c|c|c|c|c|}
\hline \multicolumn{12}{|c|}{ N2 $\mathrm{TAA}^{\#}$ (degree) } \\
\hline Baseline & $3.25 \pm 0.72$ & $3.15 \pm 0.71$ & $3.06 \pm 0.71$ & $3.25 \pm 0.73$ & 0.743 & 0.633 & 0.001 & 0.043 & 0.924 & 0.005 & 0.050 \\
\hline Follow-up & $2.81 \pm 0.69$ & $3.26 \pm 0.75$ & $3.04 \pm 0.73$ & $3.33 \pm 0.72$ & & & & & & & \\
\hline \multicolumn{12}{|c|}{ N3 TAA ${ }^{\#}$ (degree) } \\
\hline Baseline & $3.31 \pm 0.79$ & $3.02 \pm 0.97$ & $3.09 \pm 0.74$ & $3.28 \pm 0.80$ & 0.595 & 0.305 & 0.011 & 0.169 & 0.388 & $<0.001$ & 0.016 \\
\hline Follow-up & $2.84 \pm 0.81$ & $3.35 \pm 0.97$ & $3.06 \pm 0.81$ & $3.44 \pm 0.82$ & & & & & & & \\
\hline \multicolumn{12}{|c|}{ R TAA ${ }^{\#}$ (degree) } \\
\hline Baseline & $3.65 \pm 0.71$ & $3.47 \pm 0.66$ & $3.47 \pm 0.65$ & $3.50 \pm 0.62$ & 0.337 & 0.701 & 0.110 & 0.306 & 0.789 & 0.002 & 0.272 \\
\hline Follow-up & $3.25 \pm 0.68$ & $3.47 \pm 0.61$ & $3.31 \pm 0.67$ & $3.53 \pm 0.67$ & & & & & & & \\
\hline
\end{tabular}

Data are presented as group mean $\pm \mathrm{SD}$, unless otherwise stated. p-values were obtained using three-way ANCOVA adjusted for likely confounding factors of age (5-9 years), race (black, white, other), body mass index (BMI) z-score, BMI z-score change and sex. eAT: early adenotonsillectomy; WWSC: watchful waiting with supportive care; N2: stage 2 non-rapid eye movement sleep; N3: stage 3 non-rapid eye movement sleep; R: rapid eye movement sleep. \#: reported and analysed as log-transformed values. 

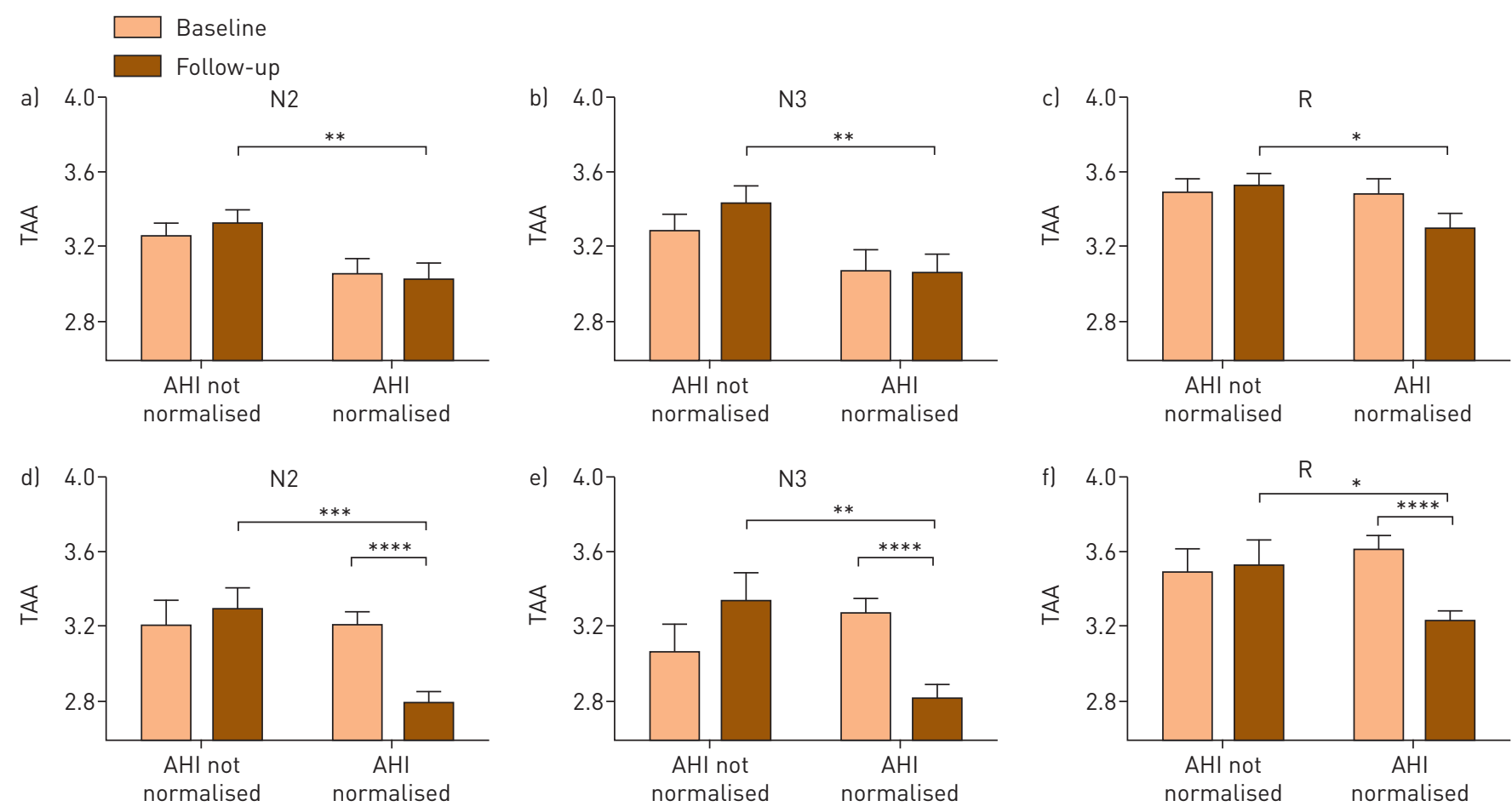

FIGURE 2 Thoraco-abdominal asynchrony (TAA) across sleep stages in children who underwent early adenotonsillectomy (eAT) (d-f) versus watchful waiting with supportive care (WWSC) (a-c) at baseline and follow-up polysomnography grouped by apnoea-hypopnoea index (AHI) normalisation. TAA values are reported and analysed as log-transformed values (degrees). Data are presented as mean \pm SEM. N2: stage 2 nonrapid eye movement sleep; N3: stage 3 non-rapid eye movement sleep; R: rapid eye movement sleep. ${ }^{*}: p<0.05 ;{ }^{* *}: p<0.01 ;{ }^{* * *}: p<0.001 ;{ }^{* * * *}$ : $p<0.0001$

a direct measure of increase in workload in terms of energy expenditure, it reflects changes in inspiratory effort due to airway obstruction [9]. Upper airway obstruction leads to increased inspiratory effort in order to maintain airway patency; this manifests as asynchronous or paradoxical inward motion of the ribcage [15-17] and hence increased TAA. TAA has been demonstrated in children with increased inspiratory effort due to upper airway obstruction and OSAS [18, 19].

Our study suggests that TAA adds important information towards the diagnosis of OSAS by quantifying overall inspiratory effort. In children with mild symptoms, in whom frank apnoeic events are rare, measuring the rate of events may not represent the full extent of respiratory disturbance during sleep. Children who snore have to overcome an increased respiratory load, but may not necessarily display frank respiratory events, desaturation or cortical arousals $[20,21]$. TAA was also associated with standard PSG measures of hypoxia (percentage of sleep time spent at $<90 \%$ oxygen desaturation) and with increased AHI throughout all stages of sleep. Presumably, children with more frequent/severe respiratory events also experience higher
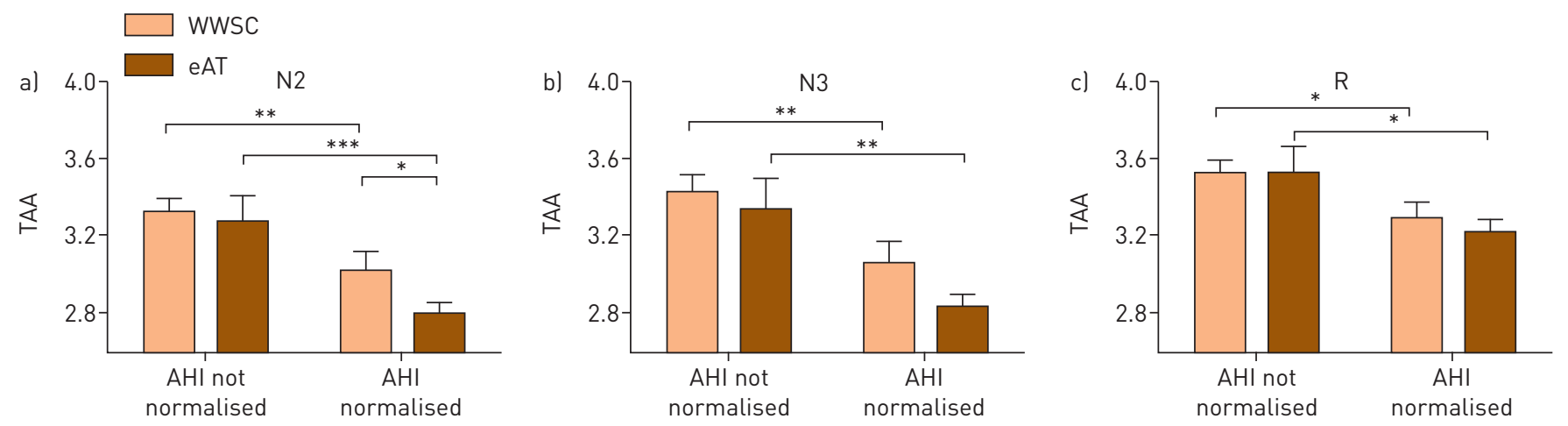

FIGURE 3 Thoraco-abdominal asynchrony (TAA) during follow-up polysomnography across sleep stages a) N2, b) N3, c) R in children who underwent early adenotonsillectomy (eAT) versus watchful waiting with supportive care (WWSC) grouped by apnoea-hypopnoea index (AHI) normalisation. TAA values are reported and analysed as log-transformed values (degrees). Data are presented as mean \pm SEM. N2: stage 2 nonrapid eye movement sleep; N3: stage 3 non-rapid eye movement sleep; R: rapid eye movement sleep. ${ }^{*}: p<0.05 ;{ }^{* *}: p<0.01 ;{ }^{* * *}: p<0.001$. 


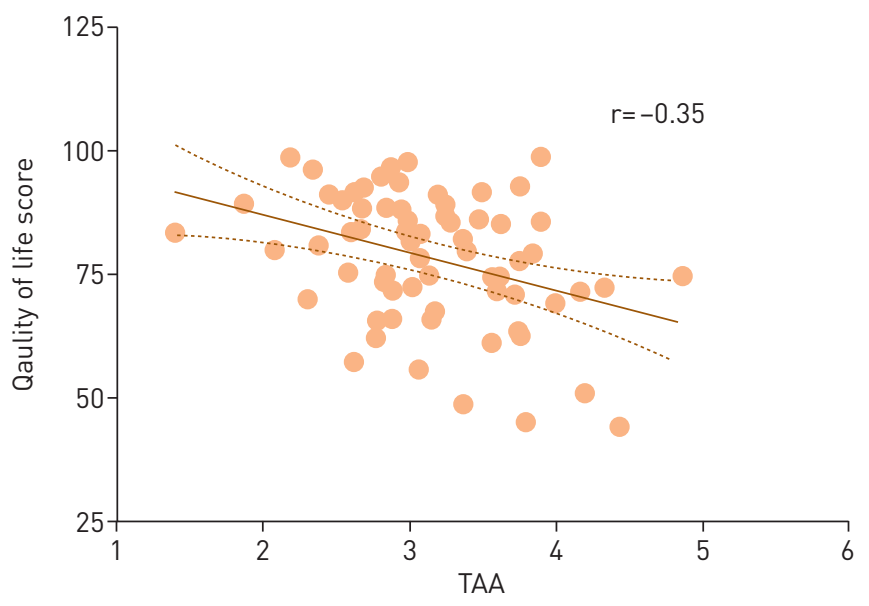

FIGURE 4 Relationship between thoraco-abdominal asynchrony (TAA) averaged across all sleep stages and Paediatric Quality of Life Inventory Parent Total Scale Score. TAA values are reported and analysed as log-transformed values (degrees). Lines indicate the linear regression function (solid) and 95\% confidence intervals (dashed).

inspiratory loads during event-free periods of sleep. While the detailed effects of increased TAA in the absence of frank apnoea or hypopnoea and underlying mechanisms have not been fully elucidated, studies in children with primary snoring have shown elevated blood pressure and reduced arterial distensibility [22, 23], subtle dysregulation of glycaemic homeostasis [24] and neurocognitive impairments [25]. Pre-pubescent rats subjected to increased upper airway loading (without hypoxia) demonstrated reduced production of growth hormone and insulin-like growth factor I and impaired longitudinal growth [26].

As has been shown previously, AT is effective at resolving clinical PSG markers of OSAS (83\% of the children in the eAT arm had AHI normalisation). However, $40 \%$ of the children in the WWSC arm, primarily those with mild OSAS, had AHI values below the clinical cut-off during follow-up, raising important questions about whom to treat and when. Our analyses show that children who underwent surgery and subsequently normalised their AHI values also demonstrated reduced TAA. This suggests a benefit of AT on inspiratory effort by enlarging the upper airway (figure 2), as the decrease in upper airway volume is related to the increase in respiratory effort during sleep [27]. Within the WWSC arm, spontaneous AHI normalisation did not coincide with TAA reduction, but children who had normal AHI values at follow-up demonstrated lower TAA at baseline, possibly because their OSAS was milder [28]. Interestingly, in children whose AHI normalised spontaneously during follow-up, TAA was inversely correlated with quality of life, suggesting that increased inspiratory effort persists in some of these children and has adverse effects on their well-being.

We have previously measured TAA during quiet, event-free sleep in children with sleep-disordered breathing undergoing AT in comparison to normal children, and observed increased TAA levels at baseline that were no longer different from TAA of normal children 6 months post AT [29], providing further evidence for the beneficial effect of AT on inspiratory effort. Our study also confirms that TAA values are higher in REM sleep than in NREM sleep [29]. This difference is possibly caused by the reduction in intercostal muscle activity, contributing to distorted ribcage movement [30] and/or decreased pharyngeal muscle activity associated with upper airway obstruction [31]. Interestingly, we observed an inverse association between TAA and age during REM sleep. Paradoxical inward ribcage motion in REM sleep and its lessening with age has been well documented in infants and toddlers [32]. Although the chest wall and ribcage are fully developed at the age of 5 years, neural respiratory control may still undergo maturation, explaining our observation [33]. Our analysis also demonstrated sex differences in TAA; to our knowledge this has not been reported previously in pre-pubescent children.

Our study has several limitations. Children in this study had only mild to moderate OSAS as defined by AHI, and the follow-up time was relatively short. More severe OSAS is likely to result in higher inspiratory effort. Although our results are based on a large randomised control trial with racially diverse groups, and included standardised measurements and high follow-up rates, there are limitations with respect to their interpretation. Several of the recordings from the original study were omitted due to poor signal quality and the per-protocol design. Sleep position is known to affect TAA [29]. We were not able to retrieve reliable information on body position from the PSG database. In addition, anti-inflammatory medication may have affected TAA in some children. 
We conclude that, in addition to its well-established effectiveness for resolving frank respiratory events in children with OSAS, AT also reduces inspiratory effort throughout quiet respiratory-event-free sleep. Surgery may therefore have an additional, previously unrecognised benefit. TAA appears to be a sensitive marker of increased inspiratory effort that is inversely associated with quality of life in children with symptomatic OSAS. Monitoring TAA over time may yield additional insight into the dynamic changes of inspiratory effort during sleep and, in combination with traditional indices of obstruction, more accurately characterise the degree of sleep-disordered breathing in children. Furthermore, our findings demonstrate that spontaneous AHI normalisation does not necessarily indicate that OSAS has resolved, highlighting the need for more sensitive measures.

\section{Acknowledgements}

We would like to thank Michael Rueschman, Division of Sleep and Circadian Disorders, Brigham and Women's Hospital, Boston, MA, USA for support with handling and interpreting the CHAT dataset.

Xiao Liu, Sarah Immanuel and Mathias Baumert had full access to all of the data in the study and take responsibility for the integrity of the data and the accuracy of the data analysis. Yvonne Pamula, James Martin and Declan Kennedy contributed substantially to the interpretation and the writing of the manuscript.

\section{References}

1 Lumeng JC, Chervin RD. Epidemiology of pediatric obstructive sleep apnea. Proc Am Thorac Soc 2008; 5: $242-252$.

2 Nisbet LC, Yiallourou SR, Walter LM, et al. Blood pressure regulation, autonomic control and sleep disordered breathing in children. Sleep Med Rev 2014; 18: 179-189.

3 Baumert M, Pamula Y, Martin J, et al. The effect of adenotonsillectomy for childhood sleep apnoea on cardiorespiratory control. ERJ Open Res 2016; 2: 00003-2016.

4 Brietzke SE, Gallagher D. The effectiveness of tonsillectomy and adenoidectomy in the treatment of pediatric obstructive sleep apnea/hypopnea syndrome: a meta-analysis. Otolaryngol Head Neck Surg 2006; 134: 979-984.

5 Bhattacharjee R, Kheirandish-Gozal L, Spruyt K, et al. Adenotonsillectomy outcomes in treatment of obstructive sleep apnea in children: a multicenter retrospective study. Am J Respir Crit Care Med 2010; 182: 676-683.

6 Costa DJ, Mitchell R. Adenotonsillectomy for obstructive sleep apnea in obese children: a meta-analysis. Otolaryngol Head Neck Surg 2009; 140: 455-460.

7 Rapoport DM. POINT: Is the apnea-hypopnea index the best way to quantify the severity of sleep-disordered breathing? Yes. Chest 2016; 149: 14-16.

8 Punjabi NM. COUNTERPOINT: Is the apnea-hypopnea index the best way to quantify the severity of sleep-disordered breathing? No. Chest 2016; 149: 16-19.

9 Hammer J, Newth C, Deakers T. Validation of the phase angle technique as an objective measure of upper airway obstruction. Pediatr Pulmonol 1995; 19: 167-173.

10 Marcus CL, Moore RH, Rosen CL, et al. A randomized trial of adenotonsillectomy for childhood sleep apnea. N Engl J Med 2013; 368: 2366-2376.

11 Redline S, Amin R, Beebe D, et al. The Childhood Adenotonsillectomy Trial (CHAT): rationale, design, and challenges of a randomized controlled trial evaluating a standard surgical procedure in a pediatric population. Sleep 2011; 34: 1509-1517.

12 Berry RB, Budhiraja R, Gottlieb DJ, et al. Rules for scoring respiratory events in sleep: update of the 2007 AASM Manual for the Scoring of Sleep and Associated Events. Deliberations of the Sleep Apnea Definitions Task Force of the American Academy of Sleep Medicine. J Clin Sleep Med 2012; 8: 597-619.

13 Redline S, Amin R, Beebe D, et al. Childhood Adenotonsillectomy Trial. National Sleep Research Resource. http://sleepdata.org/datasets/chat Date last accessed: October 14, 2016.

14 Immanuel S, Kohler M, Pamula Y, et al. Thoraco-abdominal asynchrony in children during quiet sleep using Hilbert transform. Conf Proc IEEE Eng Med Biol Soc 2012; 2012: 3448-3451.

15 Bower CM, Gungor A. Pediatric obstructive sleep apnea syndrome. Otolaryngol Clin North Am 2000; 33 : 49-75.

16 Guilleminault C, Poyares D, Palombini L, et al. Variability of respiratory effort in relation to sleep stages in normal controls and upper airway resistance syndrome patients. Sleep Med 2001; 2: 397-405.

17 Sivan Y, Ward SD, Deakers T, et al. Rib cage to abdominal asynchrony in children undergoing polygraphic sleep studies. Pediatr Pulmonol 1991; 11: 141-146.

18 Reber A, Bobbia SA, Hammer J, et al. Effect of airway opening manoeuvres on thoraco-abdominal asynchrony in anaesthetized children. Eur Respir J 2001; 17: 1239-1243.

19 Kohyama J, Shiiki T, Shimohira M, et al. Asynchronous breathing during sleep. Arch Dis Child 2001; 84: 174-177.

20 Guilleminault C, Winkle R, Korobkin R, et al. Children and nocturnal snoring: evaluation of the effects of sleep related respiratory resistive load and daytime functioning. Eur J Pediatr 1982; 139: 165-171.

21 Guilleminault C, Poyares D. Arousal and upper airway resistance (UAR). Sleep Med 2002; 3: S15-S20.

$22 \mathrm{Li} \mathrm{AM}, \mathrm{Au} \mathrm{CT}$, Ho C, et al. Blood pressure is elevated in children with primary snoring. J Pediatr 2009; 155: $362-368$.

23 Kwok KL, Ng DKK, Cheung YF. BP and arterial distensibility in children with primary snoring. Chest 2003; 123: 1561-1566.

24 Khalyfa A, Gharib SA, Kim J, et al. Peripheral blood leukocyte gene expression patterns and metabolic parameters in habitually snoring and non-snoring children with normal polysomnographic findings. Sleep 2011; 34: 153-U150.

25 Brockmann PE, Urschitz MS, Schlaud M, et al. Primary snoring in school children: prevalence and neurocognitive impairments. Sleep Breath 2012; 16: 23-29.

26 Tarasiuk A, Segev Y. Chronic upper airway resistive loading induces growth retardation via the GH/IGF-I axis in prepubescent rats. J Appl Physiol 2007; 102: 913-918. 
27 Guilleminault C, Li KK, Khramtsov A, et al. Sleep disordered breathing: surgical outcomes in prepubertal children. Laryngoscope 2004; 114: 132-137.

28 Chervin RD, Ellenberg SS, Hou XL, et al. Prognosis for spontaneous resolution of OSA in children. Chest 2015; 148: 1204-1213.

29 Immanuel SA, Kohler M, Martin J, et al. Increased thoracoabdominal asynchrony during breathing periods free of discretely scored obstructive events in children with upper airway obstruction. Sleep Breath 2015; 19: 65-71.

30 Tabachnik E, Muller NL, Bryan AC, et al. Changes in ventilation and chest wall mechanics during sleep in normal adolescents. J Appl Physiol 1981; 51: 557-564.

31 Mezzanotte WS, Tangel DJ, White DP. Influence of sleep onset on upper-airway muscle activity in apnea patients versus normal controls. Am J Respir Crit Care Med 1996; 153: 1880-1887.

32 Gaultier C, Praud JP, Canet E, et al. Paradoxical inward rib cage motion during rapid eye movement sleep in infants and young children. J Dev Physiol 1987; 9: 391-397.

33 Carroll JL, Donnelly DF. Respiratory physiology and pathophysiology during sleep. In: Sheldon SH, Kryger MH, Ferber R, Gozal D, eds. Principles and Practice of Pediatric Sleep Medicine. 2nd edn. Philadelphia, Elsevier, 2014; pp. 179-194. 